\title{
Predictive Value of Decreased Serum Estradiol Levels Following Human Chorionic Gonadotropin Trigger For Unexpected Ovulation During In Vitro Fertilization Cycle of Patients With Poor Ovarian Response
}

\section{Qianwen Huang}

Guangdong Women and Children Hospital https://orcid.org/0000-0003-1527-8534

Qianyu Zhang

Guangdong Women and Children Hospital

Li Sun

Guangdong Women and Children Hospital

\section{Ting Tang}

Guangdong Women and Children Hospital

Wenjuan Liu

Guangdong Women and Children Hospital Jie Zhang

Guangdong Women and Children Hospital

Fenghua Liu ( $\square$ liushine2016@163.com )

Guangdong Women and Children Hospital https://orcid.org/0000-0002-3860-9466

\section{Research}

Keywords: serum estradiol level, unexpected ovulation, patient with poor ovarian response, hCG trigger

Posted Date: September 2nd, 2021

DOI: https://doi.org/10.21203/rs.3.rs-845229/v1

License: (a) (1) This work is licensed under a Creative Commons Attribution 4.0 International License.

Read Full License 


\section{Abstract \\ Background}

Unexpected spontaneous ovulation typically occurs in patients with poor ovarian response (POR); however, reliable predictors are lacking. We analyzed explore the predictive value of decreased serum estradiol (E2) levels on the day after human chorionic gonadotropin (hCG) trigger during in vitro fertilization (IVF) cycles.

\section{Methods}

We retrospectively analyzed clinical data $(n=978)$ in IVF cycles between 2017 and 2020 . According to decreased ratios of E2 levels ( $\triangle E 2 \%$ ) between day 1 and day 0 of hCG trigger, patients were divided into: $\leq 10 \%$ (group A), $10-20 \%$ (group B), and $\geq 20 \%$ (group C). Basic characteristics and laboratory data were compared using analysis of variance or Mann-Whitney U-test. Logistic regression and receiver operating characteristic (ROC) curve analyses were used to assess the relationship between $\triangle E 2 \%$ and spontaneous unexpected ovulation. Fresh embryo transfer cycles in groups $A, B$, and $C$ were subgrouped as $\mathrm{A} 1, \mathrm{~B} 1$, and $\mathrm{C1}$, respectively; clinical outcomes were compared.

\section{Results}

Mean E2 and luteinizing hormone (LH) levels, ratio of premature LH peak and high progesterone levels, time interval between hCG trigger and oocyte retrieval, number of follicles $\geq 11 \mathrm{~mm}$ in diameter, and dose regimen of gonadotropin among the three groups showed significant differences $(P<0.05)$. After adjusting for relevant confounders, likelihood ratios of unexpected ovulation in groups $B$ and $C$ were 2.96 (95\% confidence interval [Cl]: 0.64-13.70), and 11.74 (95\% Cl: 3.09-44.62), respectively, compared with that in group $\mathrm{A}$. The test for trend was significant before and after correction $(P$ for trend $<0.05)$. On combining premature LH peak $(>10 \mathrm{mlU} / \mathrm{mL})$ or high progesterone level $(>1.0 \mathrm{ng} / \mathrm{mL})$ on the day of hCG, area under ROC curve (AUC) was $0.849(95 \% \mathrm{Cl}, 0.764-0.934, P<0.05)$. However, when we only considered $\triangle \mathrm{E} 2 \%$, AUC was $0.738(95 \% \mathrm{Cl}$. 0.562-0.914, $P<0.05)$. Rates of embryo implantation, clinical pregnancy, live birth in fresh embryo transfer cycle, and early abortion among the three subgroups did not differ significantly $(P>0.05)$.

\section{Conclusions}

E2 levels on the day after hCG trigger are valuable for predicting unexpected ovulation. However, their decreased levels do not directly affect clinical pregnancy outcomes.

\section{Background}


Obtaining an optimal number of high-quality oocytes through controlled ovarian stimulation (COS) without increasing the incidence of complications is critical for successful assisted pregnancy using in vitro fertilization-embryo transfer (IVF-ET) or intracytoplasmic sperm injection (ICSI) treatment cycles [1, 2]. However, few low-quality oocytes often occur in patients with poor ovarian response (POR). Improving the clinical pregnancy outcomes of patients with POR has always been a challenge in reproductive medicine. In such cases, selecting an appropriate COS protocol is an important factor.

Recently, with further understanding of the ovary stimulating drugs, the advantages of using gonadotropin releasing hormone-antagonists ( $\mathrm{GnRH}-\mathrm{A})$, such as rapid action and small dosage of gonadotropin, have been highlighted [3, 4]. Currently, it is considered that GnRH-A can be used in patients with both low- and high-ovarian responses [3]. The addition of GnRH-A can effectively inhibit the endogenous luteinizing hormone (LH) peak to prevent uncontrolled spontaneous ovulation during the COS process. However, there are approximately 2-5\% POR patients with premature LH peaks, which lead to unexpected ovulation and luteinization [5]. Therefore, it is important to identify reliable indicators for predicting unexpected ovulation. The level of serum estradiol (E2) level is an important index for evaluating follicular development during ovulation induction. When using gonadotropin to stimulate the ovaries, the serum E2 levels continue to increase with follicular growth. A slow increase or a decrease in serum E2 levels may indicate abnormal follicular growth. Therefore, considering the E2 levels at different time points of COS is particularly important. Previous studies [6-8] have focused on the relationship between E2 levels on the day of human chorionic gonadotropin (hCG) trigger and clinical pregnancy outcomes, but the data are inconclusive. Moreover, whether the change in serum E2 levels on the day after $\mathrm{hCG}$ trigger affects the occurrence of unexpected ovulation in patients with POR remains unclear.

We conducted this retrospective study to assess the predictive value of decreased serum E2 levels on the day after $\mathrm{hCG}$ trigger for unexpected ovulation and clinical outcomes during the $\mathrm{GnRH}-\mathrm{A}$ protocol in patients with POR.

\section{Methods}

\section{Study subjects}

The clinical data of 978 patients who underwent IVF/ICSI treatment were collected from the Department of Reproductive Health and Infertility, Guangdong Women and Children Hospital from January 2017 to June 2020. Among them, 295 were fresh embryo transfer cycles. The inclusion criteria for study subjects were as follows: (1) stimulation using the GnRH-A protocol, (2) patients with anti-Mullerian hormone $(\mathrm{AMH})$ levels $<1.2 \mathrm{ng} / \mathrm{mL}$ and/or the number of antral follicle count $(\mathrm{AFC})<5$, defined as POR, in accordance with the 2016 Poseidon criteria [9], and (3) decrease in E2 levels one day after hCG administration compared with that on the day of hCG administration. The exclusion criteria were as follows: (1) patients whose serum E2 levels had not been tested for various reasons, and (2) E2 level exceeding the upper limit of the instrument detection because in such a case the specific value of E2 displayed was incorrect. 


\section{Grouping method}

The subjects were divided into three groups according to the different decline rate of serum E2 level $(\triangle E 2 \%)$, which was calculated as follows: (decreased ratio of E2 levels between day 1 and day 0 of hCG trigger / E2 level on day 0 of $h C G$ trigger) $\times 100 \%$. Subjects with $\triangle E 2 \% \leq 10 \%$ were defined as group $A$, those with $\triangle E 2 \%$ between 10 and $20 \%$ were defined as group $B$, and those with $\triangle E 2 \% \geq 20 \%$ were defined as group $C$. The fresh embryo transfer cycles were divided into three subgroups: fresh embryo transfer in group A (group A1), group B (group B1), and group C (group C1).

\section{Process of assisted pregnancy}

Using the GnRH-A protocol, on days 2-3 of menstruation, gonadotropin, along with either recombinant follicle-stimulating hormone ( $r-F S H)$ or urinary FSH was administered at an initial dose ranging from 200 to $300 \mathrm{U}$. Thereafter, the gonadotropin doses were adjusted according to follicular growth until the day of hCG trigger. GnRH-A was added at $0.125-0.5 \mathrm{mg} /$ day on day 5 of gonadotropin administration or added variably according to follicular growth and hormone levels. When the dominant follicles were at least 18 $\mathrm{mm}$ in diameter, urinary hCG $(6,000-10,000 \mathrm{IU})$ or recombinant hCG $(250 \mu \mathrm{g})$ was injected as the triggering agent.

After 34-36 h of the hCG trigger, oocyte retrieval was performed. The oocytes were inseminated by IVF or ICSI according to the quality of the sperm and the clinical indication. If there were no contraindications to fresh embryo transfer, not more than two embryos were transferred on day 3 (cleavage stage) or day 5 (blastocyst stage). After transplantation, luteal support with progesterone was provided until 10 weeks after conception. Non-transferred embryos were cryopreserved using vitrification.

\section{Hormone level determination}

The blood samples of patients were collected between 7:30-9:00 AM on the start day of COS, day 0 and day 1 of hCG trigger to determine FSH, LH, E2, and progesterone levels using specific commercial kits (Roche Diagnostic Products Shanghai Co., Ltd.). The coefficient of variation between and within the batches was less than $5 \%$.

\section{Statistical indicators}

Unexpected ovulation rate was defined as the number of spontaneous ovulation cycles prior to oocyte retrieval/the total number of cycles. The rate of formation of oocyte-cumulus complexes (OCCs) was calculated as the number of oocytes retrieved divided by the number of follicles $\geq 11 \mathrm{~mm}$ in diameter on the day of hCG trigger. Clinical pregnancy was confirmed by an ultrasound evidence of a gestational sac. Clinical pregnancy rate was defined as the number of clinical pregnancy cycles divided by the number of transferred cycles. The live birth rate was defined as the number of liveborn-infant cycles divided by the number of transferred cycles.

\section{Statistical analyses}


Statistical analyses were performed using SPSS for Windows (version 26.0). Continuous variables with normal distribution are presented as the mean \pm standard deviation (SD); non-normal variables as median (interquartile range). Comparisons between groups for continuous normally distributed variables were performed using ANOVA, whereas those for nonparametric data were conducted using the MannWhitney U-test. The countable data are expressed as rate (\%) and were compared using the chi-square or Fisher's exact test. Statistical significance was set at $P<0.05$. Logistic regression analysis was used to assess the relationship between $\triangle E 2 \%$ and spontaneous unexpected ovulation, expressed as odds ratios with $95 \%$ confidence intervals ( $95 \% \mathrm{Cls}$ ). Receiver operating characteristic (ROC) curve analysis was used to calculate the area under the ROC curve (AUC), cut-off values, sensitivity, and specificity.

\section{Results}

\section{Baseline characteristics of the three groups}

No significant differences in the mean age, body mass index (BMI), baseline FSH level, AFC, and year of infertility were observed among groups $A, B$, and $C(P>0.05)$. The hormone levels of the three groups on the day of hCG trigger were compared, and the results revealed no significant difference in the mean progesterone levels $(P>0.05)$; however, the mean E2 and LH levels, ratio of premature LH peak $(>10$ $\mathrm{mlU} / \mathrm{ml})$, and ratio of high progesterone levels $(>1.0 \mathrm{ng} / \mathrm{ml})$ showed significant differences $(P<0.05)$.

Significant differences in the time interval between oocyte retrieval and hCG trigger, number of follicles $\geq$ $11 \mathrm{~mm}$ in diameter, and dosage regimen of $\mathrm{Gn}$ were observed among the three groups in comparison with those in group $C(P<0.05)$ (Table 1$)$. 
Table 1

Comparison of general basic data among groups $A, B$, and $C$.

\begin{tabular}{|c|c|c|c|c|}
\hline Characteristics & Group A $(n=585)$ & Group B $(n=235)$ & $\begin{array}{l}\text { Group } C(n= \\
158)\end{array}$ & $\begin{array}{l}P- \\
\text { value }\end{array}$ \\
\hline Age(year) & $37.77 \pm 5.10$ & $37.79 \pm 5.21$ & $38.25 \pm 4.96$ & 0.563 \\
\hline Infertility year (year) & $4.56 \pm 4.36$ & $4.17 \pm 3.79$ & $4.14 \pm 2.04$ & 0.294 \\
\hline $\mathrm{BMI}\left(\mathrm{Kg} / \mathrm{m}^{2}\right)$ & $22.07 \pm 2.85$ & $21.88 \pm 2.85$ & $21.82 \pm 2.85$ & 0.518 \\
\hline Baseline FSH (IU/L) & $10.25 \pm 5.60$ & $10.20 \pm 5.17$ & $10.43 \pm 4.95$ & 0.915 \\
\hline AFC count(n) & $3.92 \pm 2.01$ & $4.15 \pm 1.94$ & $3.87 \pm 2.23$ & 0.287 \\
\hline $\begin{array}{l}\text { The total dosages } \\
\text { of Gn (IU) }\end{array}$ & $1991.62 \pm 1115.07$ & $1990.48 \pm 1014.74$ & $\begin{array}{l}1560.36 \pm \\
1162.72\end{array}$ & 0.000 \\
\hline $\begin{array}{l}\text { The total days of } \mathrm{Gn} \\
\text { (days) }\end{array}$ & $7.95 \pm 3.15$ & $7.99 \pm 3.06$ & $6.53 \pm 4.11$ & 0.000 \\
\hline $\begin{array}{l}\text { Number of } \geq 11 \mathrm{~mm} \\
\text { follicles (n) }\end{array}$ & $3(2,4)$ & $3(1,6)$ & $4(2,7)$ & 0.000 \\
\hline $\begin{array}{l}\text { E2 levels on the day } \\
\text { of } \mathrm{hCG}(\mathrm{ng} / \mathrm{ml})\end{array}$ & $1026.5(615.85,1636.25)$ & $1005(632.5,1773.50)$ & $800(439.9,1582)$ & 0.047 \\
\hline $\begin{array}{l}\text { LH levels on the day } \\
\text { of hCG (IU/I) }\end{array}$ & $3.84(2.39,5.66)$ & $4.27(2.47,7.81)$ & $8.08(3.78,14.29)$ & 0.000 \\
\hline $\begin{array}{l}\text { P levels on the day } \\
\text { of } \mathrm{hCG}(\mathrm{ng} / \mathrm{ml})\end{array}$ & $0.54(0.33,0.8)$ & $0.60(0.32,0.8)$ & $0.56(0.31,0.96)$ & 0.352 \\
\hline $\begin{array}{l}\text { The ratio of } \\
\text { premature LH peak } \\
(\%)\end{array}$ & $6.2(36 / 585)$ & $17(40 / 235)$ & $39.2(62 / 158)$ & 0.000 \\
\hline $\begin{array}{l}\text { The ratio of } \\
\text { progesterone levels } \\
>1.0 \mathrm{ng} / \mathrm{ml}(\%)\end{array}$ & $14(82 / 585)$ & $21.3(50 / 235)$ & $22.8(36 / 158)$ & 0.006 \\
\hline The time interval (h) & $35.40 \pm 3.15$ & $34.92 \pm 4.21$ & $33.11 \pm 7.56$ & 0.000 \\
\hline
\end{tabular}

\section{Laboratory Data Of The Three Groups}

The number of oocytes retrieved, OCCs rate, unexpected ovulation rate, rate of non-retrieved oocytes, mature oocyte rate, fertilization rate, and rate of obtaining high-quality embryos showed significant differences among the three groups $(P<0.05)$. With increasing $\triangle \mathrm{E} 2 \%$, the unexpected ovulation rate of group C (8.9\%) was higher than that of group A $(0.9 \%)$ and B $(2.6 \%)(P<0.05$, Table 2$)$. 
Table 2

Comparison of laboratory data of the three groups

\begin{tabular}{|lllll|}
\hline Factors & $\begin{array}{l}\text { Group A }(\mathrm{n}= \\
\mathbf{5 8 5})\end{array}$ & $\begin{array}{l}\text { Group B }(\mathrm{n}= \\
\mathbf{2 3 5})\end{array}$ & $\begin{array}{l}\text { Group C }(\mathrm{n}= \\
\mathbf{1 5 8})\end{array}$ & $\begin{array}{l}\boldsymbol{P} \\
\text { value }\end{array}$ \\
\hline $\begin{array}{l}\text { The number of oocytes retrieved } \\
(\mathrm{n})\end{array}$ & $3(2,6)$ & $3(2,5)$ & $2(1,3)$ & 0.000 \\
\hline The ocCs rate(\%) & $84.32 \pm 24.30$ & $82.78 \pm 27.10$ & $71.89 \pm 37.55$ & 0.000 \\
\hline The premature ovulation rate (\%) & $0.9(5 / 585)$ & $2.6(6 / 235$ & $8.9(14 / 158)$ & 0.000 \\
\hline $\begin{array}{l}\text { The oocytes non-retrieved rate } \\
(\%)\end{array}$ & $3.6(21 / 585)$ & $5.5(13 / 235)$ & $16.5(26 / 158)$ & 0.000 \\
\hline The mature oocyte rate (\%) & $85.24 \pm 26.34$ & $83.37 \pm 29.69$ & $72.34 \pm 40.66$ & 0.000 \\
\hline The fertilization rate (\%) & $66.35 \pm 32.87$ & $67.11 \pm 34.79$ & $56.21 \pm 42.03$ & 0.003 \\
\hline The utilizable embryos rate (\%) & $72.99 \pm 38.88$ & $72.79 \pm 39.84$ & $55.78 \pm 46.60$ & 0.000 \\
\hline The high-quality embryo rate (\%) & $63.34 \pm 40.43$ & $63.02 \pm 40.96$ & $45.11 \pm 44.64$ & 0.000 \\
\hline
\end{tabular}

\section{Logistic regression analysis of relation between $\triangle E 2 \%$ and unexpected ovulation}

Group A was considered the reference group. The likelihood ratio of unexpected ovulation rate in groups $\mathrm{B}$ and $\mathrm{C}$ was 3.36 (95\% Cl: 0.75-5.13) and $18.86 \%$ (95\% Cl, 5.35-66.51), respectively, in comparison with that in group A. After adjusting for age; basal FSH level; AFC; gonadotropin dosage regimen; and levels of $\mathrm{E} 2, \mathrm{LH}$, and progesterone on the day of hCG trigger, and the time interval between hCG trigger and oocyte retrieval, the likelihood ratio of unexpected ovulation rate in groups B and C was 2.96 (95\% Cl: 0.64$13.70)$ and $11.74 \%$ (95\% Cl: 3.09-44.62), respectively. The test for trend was statistically significant before and after correction $(P<0.05)$.

Moreover, it was concluded that the risk of unexpected ovulation increased by 2.27 times for one standard deviation (SD) (approximately 10\%) increase in $\triangle \mathrm{E} 2 \%(95 \% \mathrm{Cl}: 1.76-2.94)$. After adjusting relevant confounders, the risk of unexpected ovulation increased by 2.22 times (95\% Cl: 1.72-2.87) (Table 3). 
Table 3

Logistic regression analysis of relation between $\triangle E 2 \%$ and unexpected ovulation

\begin{tabular}{|lll|}
\hline Factors & Odds ratio $(95 \% \mathrm{Cl})$ & Odds ratio-adjusted $(95 \% \mathrm{Cl})$ \\
\hline Group $\mathrm{A}(\triangle \mathrm{E} 2 \%<10 \%)$ & Ref & Ref \\
\hline Group $\mathrm{B}(10 \%>\triangle \mathrm{E} 2 \%>20 \%)$ & $3.36(0.75-15.13)$ & $2.96(0.64-13.70)$ \\
\hline Group $\mathrm{C}(\triangle \mathrm{E} 2 \% \bigotimes 20 \%)$ & $18.86(5.35-66.51)$ & $11.74(3.09-44.62)$ \\
\hline$P$ for trend & 0.000 & 0.000 \\
\hline Per $10 \%(1 \mathrm{SD})$ decrement & $2.27(1.76-2.94)$ & $2.22(1.72-2.87)$ \\
\hline
\end{tabular}

\section{ROC curve analysis of predictive value of $\triangle E 2 \%$ for unexpected ovulation}

To assess the predictive value of $\triangle E 2 \%$ for unexpected ovulation, ROC curves were generated. When we combined premature LH peak $(>10 \mathrm{mIU} / \mathrm{ml})$ or high progesterone level $(>1.0 \mathrm{ng} / \mathrm{ml})$ on the day of hCG trigger, the AUC was 0.849 (95\% Cl: $0.764-0.934, P<0.05)$, and the maximum Youden Index was 0.610 , with a cut-off of 0.260 (Fig. 1). However, when we considered only $\triangle \mathrm{E} 2 \%$, the AUC was $0.738(95 \% \mathrm{Cl}$ : $0.562-0.914, P<0.05$ ), and the maximum Youden Index was 0.380 , with a cut-off of 0.072 (Fig. 2). Therefore, the results suggest that the $\triangle \mathrm{E} 2 \%>26 \%$ in cycles with premature LH peak and high progesterone levels on the day of hCG trigger is a valuable predictor of unexpected ovulation.

\section{Clinical Data Of Subgroups A1,b1, And C1}

A total of 295 cases of fresh embryo transfer were performed in the study data, comprising 189 cases of fresh embryo transfer cycle in group A (marked as group A1), 79 cases in group B (marked as group B1), and 27 cases in group C (marked as group C1). Comparison of the baseline characteristics and clinical outcomes of the three subgroups showed no significant difference in mean age, years of infertility, BMI, baseline FSH level, AFC counts, and the total dosages of gonadotropin $(P>0.05)$. The three subgroups were balanced and comparable. There was no significant difference in the rates of embryo implantation, clinical pregnancy, live birth in fresh embryo transfer cycle, and early abortion among the three subgroups $(P>0.05)$ (Table 4). 
Table 4

Comparison of baseline characteristics and clinical outcomes among subgroups A1, B1, and C1.

\begin{tabular}{|lllll|}
\hline Factors & $\begin{array}{l}\text { Group A1 }(\mathbf{n}= \\
\text { 189) }\end{array}$ & $\begin{array}{l}\text { Group B1 }(\mathbf{n}= \\
\text { 79) }\end{array}$ & $\begin{array}{l}\text { Group C1 }(\mathbf{n}= \\
\mathbf{2 7})\end{array}$ & $\begin{array}{l}\boldsymbol{P} \\
\text { value }\end{array}$ \\
\hline Age(year) & $36.73 \pm 5.06$ & $35.65 \pm 5.26$ & $36.85 \pm 5.05$ & 0.262 \\
\hline Infertility year (year) & $4.40 \pm 3.90$ & $3.74 \pm 2.97$ & $4.30 \pm 2.51$ & 0.389 \\
\hline BMI (Kg/m²) & $21.83 \pm 2.60$ & $21.10 \pm 2.52$ & $21.84 \pm 3.01$ & 0.107 \\
\hline Baseline FSH (IU/L) & $8.76 \pm 3.70$ & $8.79 \pm 3.34$ & $8.75 \pm 3.58$ & 0.959 \\
\hline AFC count(n) & $4.49 \pm 1.88$ & $4.77 \pm 1.98$ & $4.26 \pm 2.18$ & 0.396 \\
\hline The total dosages of Gn (IU) & $2621.30 \pm$ & $2572.78 \pm$ & $2217.11 \pm$ & 0.088 \\
\hline Endometrium thickness on day of & 877.70 & 715.20 & 891.91 & 0.571 \\
\hline ET (mm) & $9.83 \pm 2.10$ & $9.72 \pm 2.10$ & $9.38 \pm 1.65$ & \\
\hline Embryo transfer number (n) & $1.83 \pm 0.42$ & $1.80 \pm 0.46$ & $1.82 \pm 0.42$ & 0.783 \\
\hline Embryo implantation rate (\%) & $27.8(97 / 349)$ & $33.1(47 / 142)$ & $26.0(13 / 50)$ & 0.444 \\
\hline Clinical Pregnancy rate (\%) & $42.3(80 / 189)$ & $43.0(34 / 79)$ & $37.0(10 / 27)$ & 0.854 \\
\hline The early abortion rate (\%) & $21.3(17 / 80)$ & $17.6(6 / 34)$ & $30.0(3 / 10)$ & 0.697 \\
\hline Live birth rate (\%) & $29.1(55 / 189)$ & $32.9(26 / 79)$ & $18.5(5 / 27)$ & 0.364 \\
\hline ET: embryo transfer. & & & & \\
\hline
\end{tabular}

\section{Discussion}

Serum E2 levels play a vital role in follicular growth, oocyte maturation, embryonic development, and endometrial receptivity. In the present study, we selected subjects who demonstrated POR. The primary concern was the availability of sufficient and high-quality oocytes and embryos. The results showed that the decrease in E2 levels on the day after hCG trigger was a valuable predictor of unexpected ovulation in patients with POR. However, the decline in E2 levels may not directly affect the clinical outcomes of fresh embryo transfer in IVF/ICSI.

Although previous studies [10-12] have focused on the effect of serum E2 levels or progesterone / E2 ratios on the day of hCG trigger on clinical outcomes, there was a lack of consensus in this regard. Two high-quality studies $[6,13]$ concluded that the E2 levels on the day of hCG trigger had no significant predictive value for the occurrence of premature ovulation in the natural cycle; however, the ratio of E2 change between the day 0 and 1 of hCG administration was significant. Another study [14] found that decreased E2 levels on the day after hCG trigger was an effective prediction index for premature ovulation in the natural cycle. Similar to the literature, the present study not only focused on E2 levels on the day of 
hCG, but also on the change in the ratio of E2 levels between day 0 and 1 of hCG trigger. However, the novelty of this study is that it focuses on the decreased ratios of E2 levels on the day after hCG trigger; helping us to predict the occurrence of unexpected spontaneous ovulation. The decrease in E2 levels at any time point will make clinicians vigilant, but the corresponding measures to be realized are still based on clinical experience. In the present study, it was observed that the higher the ratio of decreased E2 levels on the day after hCG trigger, the higher the incidence rate of unexpected ovulation and lower the number of oocytes, OCCs, mature oocytes, fertilization, and high-quality embryos obtained. We further explored the relationship between the different decreased rates of E2 level following hCG trigger and the incidence of premature ovulation. After adjusting for relevant confounders, the risk of unexpected ovulation by a $30 \%$ decrease in E2 level was 11.72 times higher than that of a $10 \%$ decrease; and the trend was statistically significant. Moreover, when the decreased ratios of E2 were standardized, it was found that the risk of unexpected ovulation increased by 2.22 times for each SD (approximately 10\%) of decreased E2 on the day after hCG administration. Therefore, the decrease in E2 levels on the day after hCG trigger is an effective indicator for predicting premature ovulation.

Increase in the LH peak and/or increased progesterone levels on the day of hCG trigger are currently effective indicators for predicting premature ovulation. However, these studies are not unified in their conclusions. Most studies [15-17] suggest that with LH level $\geq 10 \mathrm{mlU} / \mathrm{mL}$ and progesterone levels $>1.0$ $\mathrm{ng} / \mathrm{mL}$ on the day of hCG trigger, the probability of premature ovulation increases. Effect of the GnRH-A protocol is similar to that of the $\mathrm{GnRH}$ agonist protocol in controlling the early onset of the LH peak [18, 19]. However, in the elderly and patients with POR, the LH peak is still likely to occur, resulting in early ovulation. This is because the secretion of gonadotrophin peak-attenuating factor (GnSAF), the key factor for controlling the LH peak to prevent unexpected ovulation, is reduced in patients with POR and in elderly women. The results of this study showed that higher the decreased ratio of E2 level on the day after hCG trigger, higher the LH levels on the day of hCG trigger and thus, higher the ratio of early LH peak and early rise in progesterone levels. The ROC curve analysis suggested that combination of $\mathrm{LH}$ and progesterone levels on the day of hCG trigger, and the decreased E2 levels were more valuable in predicting unexpected ovulation.

Previous studies on whether the fluctuation of E2 level influenced the clinical outcomes were discordant. Some studies $[6,7,13]$ suggested that the E2 level on the day of hCG trigger affected clinical pregnancy rates. However, most studies [20-22] suggested that E2 levels before oocyte retrieval cannot be used as the sole predictor of clinical outcomes. The different conclusions of these studies could also be related to the different research groups and COS protocols. In this study, we focused on whether the clinical outcomes of patients with POR were affected by the different decline rates of E2 levels on the day after the hCG trigger in the GnRH-A protocol. Our results showed that there was no significant difference in the clinical or live birth rates. The main reason for this is the stringent assessment of the appropriate conditions for fresh embryo transfer. If observations such as abnormal progesterone levels, endometrial abnormalities, or abnormal uterine environment are conducted, the whole embryo freezing method will be opted. We also found that the basic characteristics of the three subgroups were balanced, which is one of the reasons for the lack of significant differences in clinical outcomes. Therefore, these results further 
support the idea that the decrease in E2 level on the day after hCG trigger cannot be used as the sole predictor of clinical outcome.

Lu et al. [14] found that when E2 decreased after hCG administration, emergency oocyte retrieval could effectively reduce the incidence of premature ovulation and the cancelation rate; in particular, emergency oocyte retrieval did not reduce the rate of mature oocytes and implantation rate. As shown in Table 1, it is suggested that more the level of E2 decreased, shorter the time interval between hCG trigger and oocyte retrieval. This is related to active emergency oocyte retrieval to improve the oocyte retrieval rate in clinical practice. Nevertheless, there are a few limitations to this study. Briefly, we failed to further explore the exact relationship between the rate of E2 decline on the next day of hCG trigger and the timing of oocyte retrieval, and, currently, there is not enough literature on the same. Therefore, further randomized controlled studies are required to provide valuable information for clinical practice.

\section{Conclusions}

In summary, we explored the predictive value of E2 decline on the day after hCG trigger in unexpected spontaneous ovulation, and found that the higher the E2 decline, the greater was the incidence of premature ovulation. Moreover, combination of the LH or progesterone levels on the day of hCG trigger, and the E2 level decrease on the day after hCG trigger can better predict unexpected spontaneous ovulation, to ensure effective intervention measures and guide clinical work. However, the decreased ratios of E2 levels on the day after hCG trigger did not directly affect the clinical pregnancy outcome.

\section{List Of Abbreviations}

$\triangle \mathrm{E} 2 \%$ - decline rate of serum $\mathrm{E} 2$ level

AFC - antral follicle count

$\mathrm{AMH}$ - anti-Mullerian hormone

AUC - area under the ROC curve

$\mathrm{BMI}$ - body mass index

$\mathrm{Cl}$ - confidence intervals

COS - controlled ovarian stimulation

E2 - estradiol

FSH - follicle-stimulating hormone

GnRH-A - gonadotropin-releasing hormone antagonist 
hCG - human chorionic gonadotropin

ICSI - intracytoplasmic sperm injection

IVF-ET - in vitro fertilization-embryo transfer

LH - luteinizing hormone

ROC - Receiver operating characteristic

SD - standard deviation

\section{Declarations}

\section{Ethics approval and consent to participate}

This study was approved by the Ethics Committee of Guangdong Women and Children Hospital and has been performed in accordance with the principles of Declaration of Helsinki.

\section{Consent for publication}

Not applicable.

\section{Availability of data and material}

Not applicable.

\section{Competing interests}

None declared.

\section{Funding}

The whole study was supported by Natural Science Foundation of Guangdong Province (No. 2016A030313817).

\section{Authors' contributions}

Q.H, Q.Z and L. S were responsible for experiment conception, collection, and analysis of data. W.L, T. T and $\mathrm{J}$. $Z$ were responsible for experiment design, experiment conception and manuscript writing. F.L. designed the work, provided technical guidance and final approval of the manuscript. All authors read and approved the final manuscript.

\section{Acknowledgments}

We thank Editage (www.editage.cn) for English language editing. 


\section{References}

1. Malchau SS, Henningsen AA, Forman J, Loft A, Nyboe Andersen A, Pinborg A. Cumulative live birth rate prognosis based on the number of aspirated oocytes in previous ART cycles. Human reproduction (Oxford. England). 2019;34:171-80.

2. Magnusson $\AA$, Källen $K$, Thurin-Kjellberg A, Bergh $C$. The number of oocytes retrieved during IVF: a balance between efficacy and safety. Human reproduction (Oxford. England). 2018;33:58-64.

3. Al-Inany HG, Youssef MA, Ayeleke RO, Brown J, Lam WS, Broekmans FJ. Gonadotrophin-releasing hormone antagonists for assisted reproductive technology. Cochrane Database Syst Rev. 2016;4:Cd001750.

4. Lambalk CB, Banga FR, Huirne JA, Toftager M, Pinborg A, Homburg R, et al. GnRH antagonist versus long agonist protocols in IVF: a systematic review and meta-analysis accounting for patient type. Hum Reprod Update. 2017;23:560-79.

5. Martinez F, Barri PN, Coroleu B, Tur R, Sorsa-Leslie T, Harris WJ, et al. Women with poor response to IVF have lowered circulating gonadotrophin surge-attenuating factor (GnSAF) bioactivity during spontaneous and stimulated cycles. Human reproduction (Oxford England). 2002;17:634-40.

6. Reljic M, Vlaisavljevic V, Gavric V, Kovacic B, Cizek-Sajko M. Value of the serum estradiol level on the day of human chorionic gonadotropin injection and on the day after in predicting the outcome in natural in vitro fertilization/intracytoplasmic sperm injection cycles. Fertility sterility. 2001;75:53943.

7. Kondapalli LA, Molinaro TA, Sammel MD, Dokras A. A decrease in serum estradiol levels after human chorionic gonadotrophin administration predicts significantly lower clinical pregnancy and live birth rates in in vitro fertilization cycles. Human reproduction (Oxford. England). 2012;27:2690-7.

8. Huang J, Lu X, Lin J, Wang N, Lyu Q, Gao H, et al. A Higher Estradiol Rise After Dual Trigger in Progestin-Primed Ovarian Stimulation Is Associated With a Lower Oocyte and Mature Oocyte Yield in Normal Responders. Front Endocrinol. 2019;10:696.

9. Alviggi C, Andersen CY, Buehler K, Conforti A, De Placido G, Esteves SC, et al. A new more detailed stratification of low responders to ovarian stimulation: from a poor ovarian response to a low prognosis concept. Fertility sterility. 2016;105:1452-3.

10. Kosmas IP, Kolibianakis EM, Devroey P. Association of estradiol levels on the day of hCG administration and pregnancy achievement in IVF: a systematic review. Human reproduction (Oxford England). 2004;19:2446-53.

11. Kyrou D, Popovic-Todorovic B, Fatemi HM, Bourgain C, Haentjens P, Van Landuyt L, et al. Does the estradiol level on the day of human chorionic gonadotrophin administration have an impact on pregnancy rates in patients treated with rec-FSH/GnRH antagonist? Human reproduction (Oxford. England). 2009;24:2902-9.

12. Li X, Zeng C, Shang J, Wang S, Gao XL, Xue Q. Association between serum estradiol level on the human chorionic gonadotrophin administration day and clinical outcome. Chin Med J. 
2019;132:1194-201.

13. Reljic M, Vlaisavljević V. The preovulatory serum estradiol pattern in natural IVF/ICSI cycles. J Assist Reprod Genet. 1999;16:535-9.

14. Lu X, Khor S, Zhu Q, Sun L, Wang Y, Chen Q, et al. Decrease in preovulatory serum estradiol is a valuable marker for predicting premature ovulation in natural/unstimulated in vitro fertilization cycle. Journal of ovarian research. 2018;11:96.

15. Group EaMEOS. Comparable clinical outcome using the GnRH antagonist ganirelix or a long protocol of the $\mathrm{GnRH}$ agonist triptorelin for the prevention of premature LH surges in women undergoing ovarian stimulation. Human reproduction (Oxford England). 2001;16:644-51.

16. Engel JB, Ludwig M, Felberbaum R, Albano C, Devroey P, Diedrich K: Use of cetrorelix in combination with clomiphene citrate and gonadotrophins: a suitable approach to 'friendly IVF'? Human reproduction (Oxford, England). 2002;17:2022-6.

17. Lambalk CB, Leader A, Olivennes F, Fluker MR, Andersen AN, Ingerslev J, et al. Treatment with the $\mathrm{GnRH}$ antagonist ganirelix prevents premature $\mathrm{LH}$ rises and luteinization in stimulated intrauterine insemination: results of a double-blind, placebo-controlled, multicentre trial. Human reproduction (Oxford England). 2006;21:632-9.

18. Reichman DE, Zakarin L, Chao K, Meyer L, Davis OK, Rosenwaks Z. Diminished ovarian reserve is the predominant risk factor for gonadotropin-releasing hormone antagonist failure resulting in breakthrough luteinizing hormone surges in in vitro fertilization cycles. Fertility sterility. 2014;102:99-102.

19. Kochhar PK, Ghosh P. Diminished Ovarian Reserve Predisposes to Premature Luteinizing Hormone Surges in Gonadotropin-Releasing Hormone Antagonist Cycles in In vitro Fertilization. Journal of human reproductive sciences. 2020;13:191-5.

20. Chiasson MD, Bates GW, Robinson RD, Arthur NJ, Propst AM. Measuring estradiol levels after human chorionic gonadotropin administration for in vitro fertilization is not clinically useful. Fertility sterility. 2007;87:448-50.

21. Meyer WR, Beyler SA, Baker ST, Somkuti SG, Lowden DA, Grainger DA. Value of estradiol response after human chorionic gonadotropin administration in predicting in vitro fertilization success. Fertility sterility. 1999;72:542-5.

22. Huang R, Fang C, Wang N, Li L, Yi Y, Liang X. Serum estradiol level change after human chorionic gonadotropin administration had no correlation with live birth rate in IVF cycles. Eur J Obstet Gynecol Reprod Biol. 2014;178:177-82.

\section{Figures}




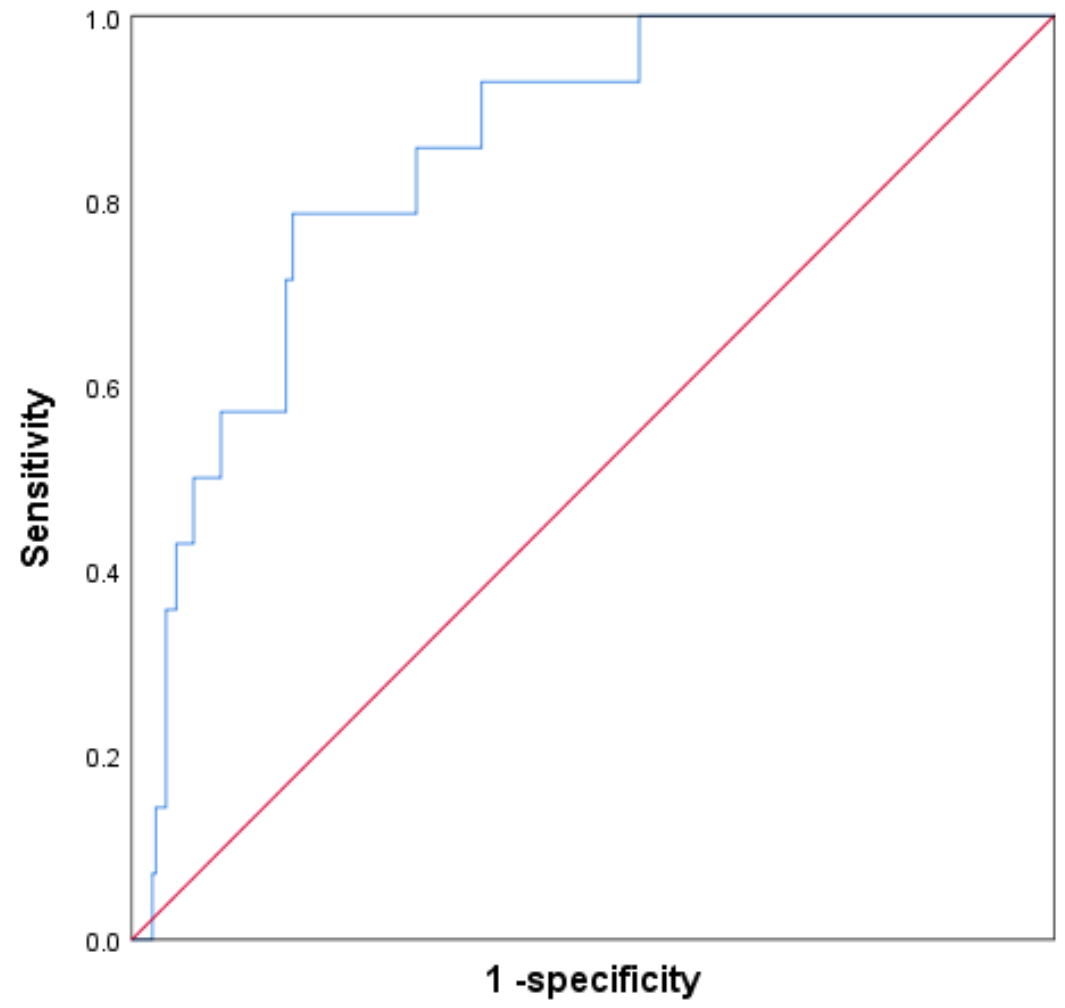

Figure 1

ROC curve analysis of predictive value of $\triangle \mathrm{E} 2 \%$ for unexpected ovulation, which combined premature $\mathrm{LH}$ peak $(>10 \mathrm{mlU} / \mathrm{ml})$ or the high P level $(>1.0 \mathrm{ng} / \mathrm{ml})$ on the day of $\mathrm{hCG}$. 


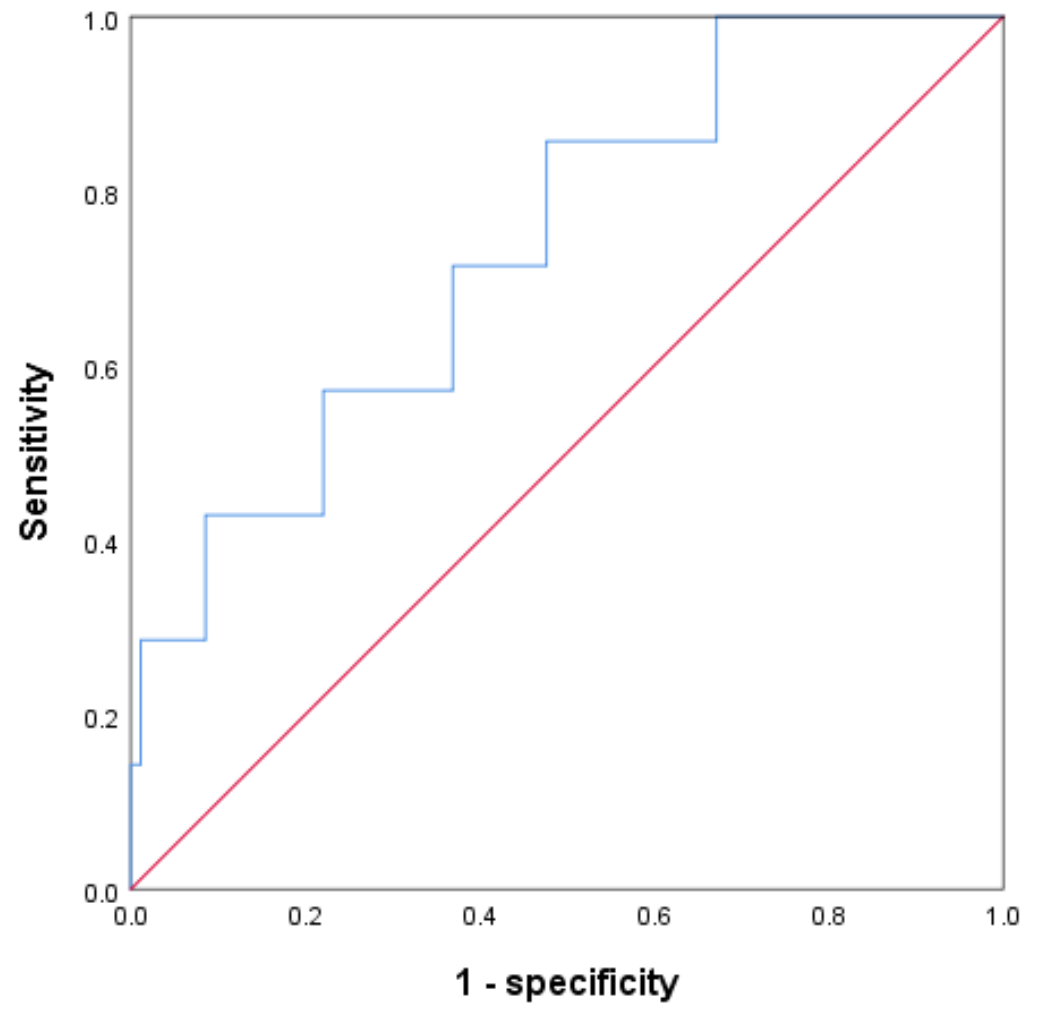

Figure 2

ROC curve analysis of predictive value of $\triangle E 2 \%$ for unexpected ovulation. 